\title{
Automatic color correction: a region-based approach and performance evaluation using full reference metrics
}

\author{
Dieu Sang Ly, ${ }^{\text {a }}$ Serge Beucher, ${ }^{a}$ Michel Bilodeau, ${ }^{a}$ Stelian Persa,b Klaas Jan Damstra,b \\ Robert Pot, ${ }^{\text {b Jan Van Rooy, }}{ }^{\text {b }}$ \\ ${ }^{a}$ MINES ParisTech, PSL - Research University, CMM - Centre for mathematical morphology, 35 rue St Honoré, \\ 77300 Fontainebleau, France

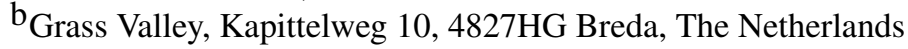

\begin{abstract}
This article proposes a solution to automatic color correction between two images/videos based on region correspondences. It starts with image segmentation by marker-controlled watershed transformation, which is faster and produces more uniform regions with better adherence to object boundaries than the segmentation in previous color correction approaches. Then, regions between two images are matched using point feature correspondences which are invariant to geometric transformation and illumination change. Finally, the color distorted image is corrected using the color statistics of corresponding regions and the color transfer functions weighted by influence masks. We demonstrate the experimental results using several data sets and evaluate the color correction by different measures of image similarity.
\end{abstract}

Keywords: color correction, color transfer, region segmentation, region matching, color similarity, image quality.

Address all correspondence to: Dieu Sang Ly; E-mail: lydieusang@gmail.com

\section{Introduction}

Color correction refers to modifying the color of an input image (color distorted image) so that it is similar to the color of a reference image. Color correction methods can be classified into two categories: one based on pixel correspondences ${ }^{1}$ and the other based on color statistics ${ }^{2] 3}$ The first one relies on correspondence accuracy whereas the second one does not require exact correspondences. There are two main groups of statistical approaches: histogram matching and color distribution modification. The basic histogram matching method computes a mapping that aligns the histograms of two images $\stackrel{4}{4}^{\text {It }}$ assumes that both images are captured from the same viewpoint and under the same illumination. In order to deal with situations when these strong assumptions are violated, Kagarlitsky et a ${ }^{[5}$ used the histogram matching as the building block and proposed several solutions based on the computation of consistent color mappings. The second group of statistical approaches utilizes the color transfer function to scale and shift the color distribution of 
the input image towards the reference one. The global color correction ${ }^{2}$ computes the parameters of the global color transfer from the entire images and hence can produce correct results uniquely when two cameras observe the same scenes under the same illumination. On the other hand, the local color correction ${ }^{67} 7$ applies local color transfer functions to different region pairs.

Most of the local (or region-based) approaches consist of three steps: region segmentation, region matching to compute the color mappings and color transfer. According to a survey of color correction algorithms in, $\frac{8}{6}$ the region-based approach proposed by Tai et al ${ }^{6}$ produced the best results among several compared approaches. First, both images are segmented by ExpectationMaximization (EM) algorithm and characterized by the Gaussian Mixture Model, where each region is associated with a Gaussian component. Then, regions between two images are matched based on their Gaussian mean value or their overlapping rate. Finally, the color-corrected image is produced by combining the color transfer functions applied to all region pairs.

Concerning the region segmentation, Oliver et al ${ }^{7}$ proposed an improvement of the previous work in ${ }^{6}$ by using the Mean-Shift algorithm which is less time-consuming than the EM algorithm and does not require the predefined number of regions as the EM algorithm does. In this work, we propose to decompose the images into regions using watershed transformation, which is not only unsupervised but also much less time-consuming than the Mean-Shift segmentation. For instance, given a 960x540 image as illustrated in figure 1 - left, it takes $55 \mathrm{msec}$. by watershed transform (plus $1 \mathrm{sec}$. by region fusion) and $27 \mathrm{sec}$. by Mean-Shift. In addition, it can be seen that the Mean-Shift algorithm does not return uniform regions with a good adherence to region boundaries as the watershed transform does. In order to suppress the redundant details and sharpen the region contours in Mean-Shift segmentation, we may increase the spatial and color range parameters, and consequently augment the computation time dramatically. 

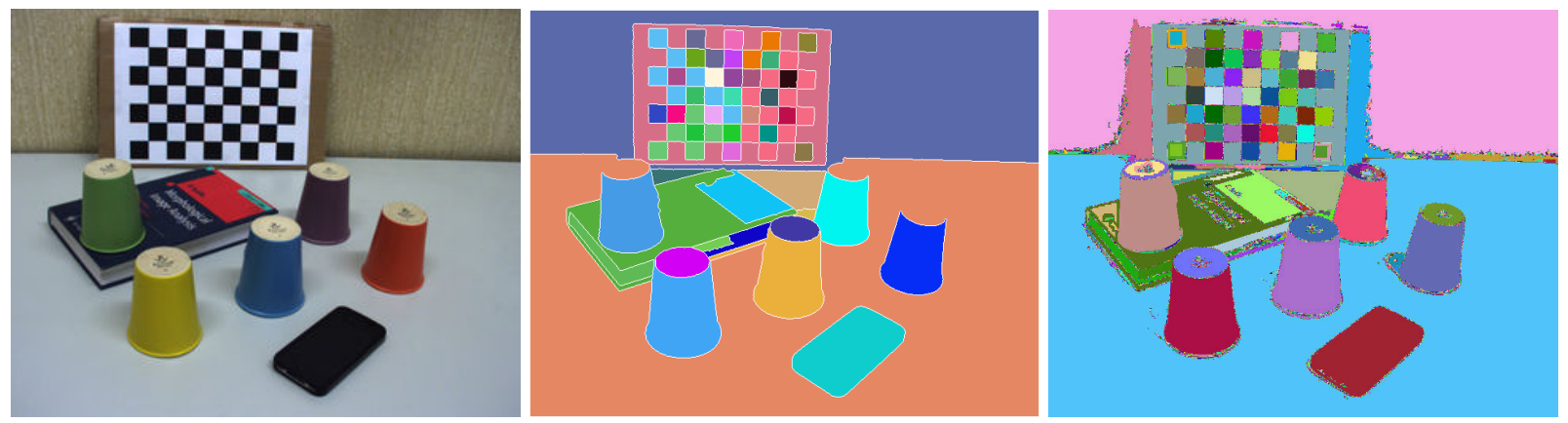

Fig 1 Segmentation of the left image by the watershed transformation (middle) and the Mean-Shift algorithm (right)

Regarding the region matching, the first solution is based on region similarity, i.e. pairing regions using their color (as in ${ }^{6}$ ), location, area or other characteristics. On one hand, using color as matching criterion assumes that the color of two regions be sufficiently similar, therefore is irrelevant in color correction application. On the other hand, location and area criteria can fail in case of complex transformations between two images, for example significant zooming or translation. Figure 2- middle shows the region matching between two segmented images on the left using their region color and location. First, the region color is calculated from the average of the color of all region pixels in LAB space. The region location is assumed to be at the region centroid. Next, given a threshold of region location, we search, in the neighborhood of each region of the input image, the region of the reference image having the closest color. As the depth of the scene is significant in these two images, the regions distant from the cameras have a remarkable translation between two images whereas the regions close to the cameras do not. Consequently, the correspondences are erroneous when we use a single threshold of region location. In other words, searching region correspondences based on location criterion is not robust to image transformation. Moreover, the color of these two images is so different that using the color in region matching produces incorrect result. The second solution assumes known image registration. ${ }^{7}$ Each region in the segmented input image is mapped to the unsegmented reference image using the 
pre-computed image transformation and the overlaid region is considered as its match. The first drawback of this method is that it requires coarsely registered images. In particular, the authors assume that the input image is entirely included in the reference image to facilitate the image registration. This strong assumption rarely holds when two cameras have random field of view. In case of complex image transformations, it is impossible to map all regions from one image to the other due to the extrapolation problem as a transformation can correctly map the image region straddled by points used to compute that transformation and is less accurate with distance from this region. 9 Figure 2- right presents the mapping of regions from the input image to the reference one. Even if point correspondences are well distributed over the images, the transformation still can handle only regions straddled by the most accurate matched points. The second drawback of this method is that the region projection will result in wrong matches in case of occultation: if a region of the input image is mapped to an occult region of the reference image, the computation of the color of this occult region is incorrect. As a consequence, the parameters of the color transfer computed from this region are wrong. In this work, we present a region matching process based on point feature correspondences, which is independent of image registration and able to handle images under different acquisition conditions.

This article proposes an approach of local color correction following the aforementioned framework with the main contributions as follows: first, the region segmentation is realized by the marker-controlled watershed transformation, which is much faster and produces more uniform regions with better adherence to region boundaries than the segmentation in previous color correction methods. Next, the region pairing is leveraged by point feature correspondences, which are invariant to geometric transformation and illumination change. Lastly, the input image is corrected using the color transfer functions of all region matches weighted by influence masks. We propose 

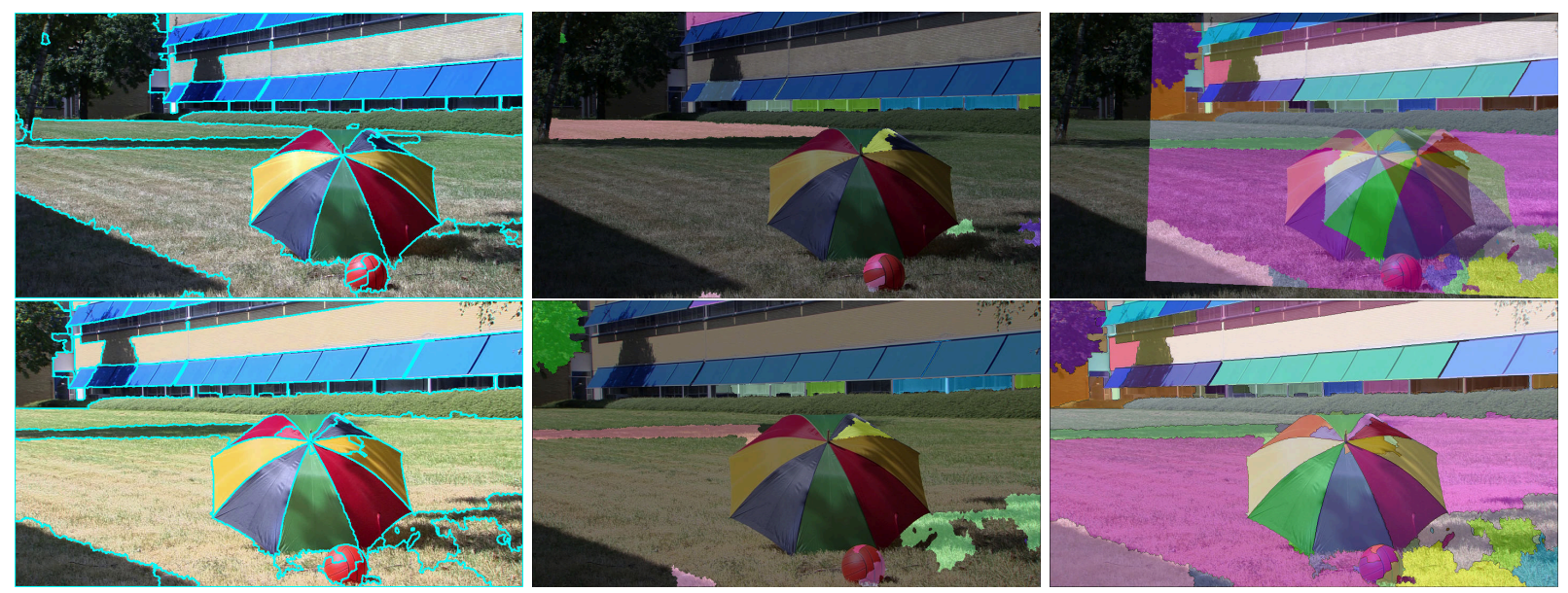

Fig 2 Region matching based on region color, location or known image registration. Left: reference (upper) and input (lower) images with region segmentation. Middle: matching by region color and location. Matched regions are displayed with the same color. Right: matching by region mapping from the input to the reference images. Each segmented region of the input image is illustrated by a color. The mapping is uniquely correct around the building.

to normalize the color distances in the influence masks to ensure that the weights are comparable among different region color ranges. This approach has been previously published in. ${ }^{[10}$ Besides developing some technical aspects in more details, we introduce the region fusion algorithm after the region segmentation step and assess the color correction results using several measurements. The rest of this paper is organized as follows: section 2 describes our proposed approach; section 3 presents some experimental results evaluated by different image quality metrics and section 4 concludes the paper.

\section{Region-based color correction}

The local (region-based) color correction approach we present in this article is composed of three tasks: segment both input (color distorted) and reference images into regions, search for region correspondences between these images and apply the color transfer to the color distorted image. 


\subsection{Region segmentation}

The original images are segmented into regions using watershed transformation.11 The idea is to consider a gray-scale image as a topographic relief and to flood this relief from different sources until they start to merge. This results in watershed lines separating different catchment basins. In addition, predefined markers can be used as flooding sources to control the segmentation, e.g. to avoid over segmentation. The marker-controlled watershed segmentation is as follows

a. Computation of segmentation criterion and markers: In order to partition an image into homogeneous regions, we can use the image gradient as the segmentation criterion (or the aforementioned topographic relief) since the gradient value is low within a homogeneous region and high at its boundary. The markers should be located inside the regions, hence can be computed from the local minima of the gradient image or by applying a threshold to the gradient image. Note that we compute the gradient from color image by taking the supremum of the gradients of all color channels. The gradient from all color channels preserves region boundaries better than the gradient from gray-level image, as illustrated in figure 3 .

b. Marker-controlled watershed segmentation: The image gradient and markers are provided to watershed segmentation. If the resulting regions are more numerous than expected, we can run an additional region fusion, which is summarized in algorithm 1 . If the color difference between two adjacent regions is inferior to a given threshold, the BoundaryEliminator operator eliminates their inner boundary and keeps their outer boundaries with other regions in order to avoid incorrect boundary elimination and region fusion. Figure 4 shows two images captured by two cameras with different photometric parameters and their watershed segmentation followed by region fusion. 


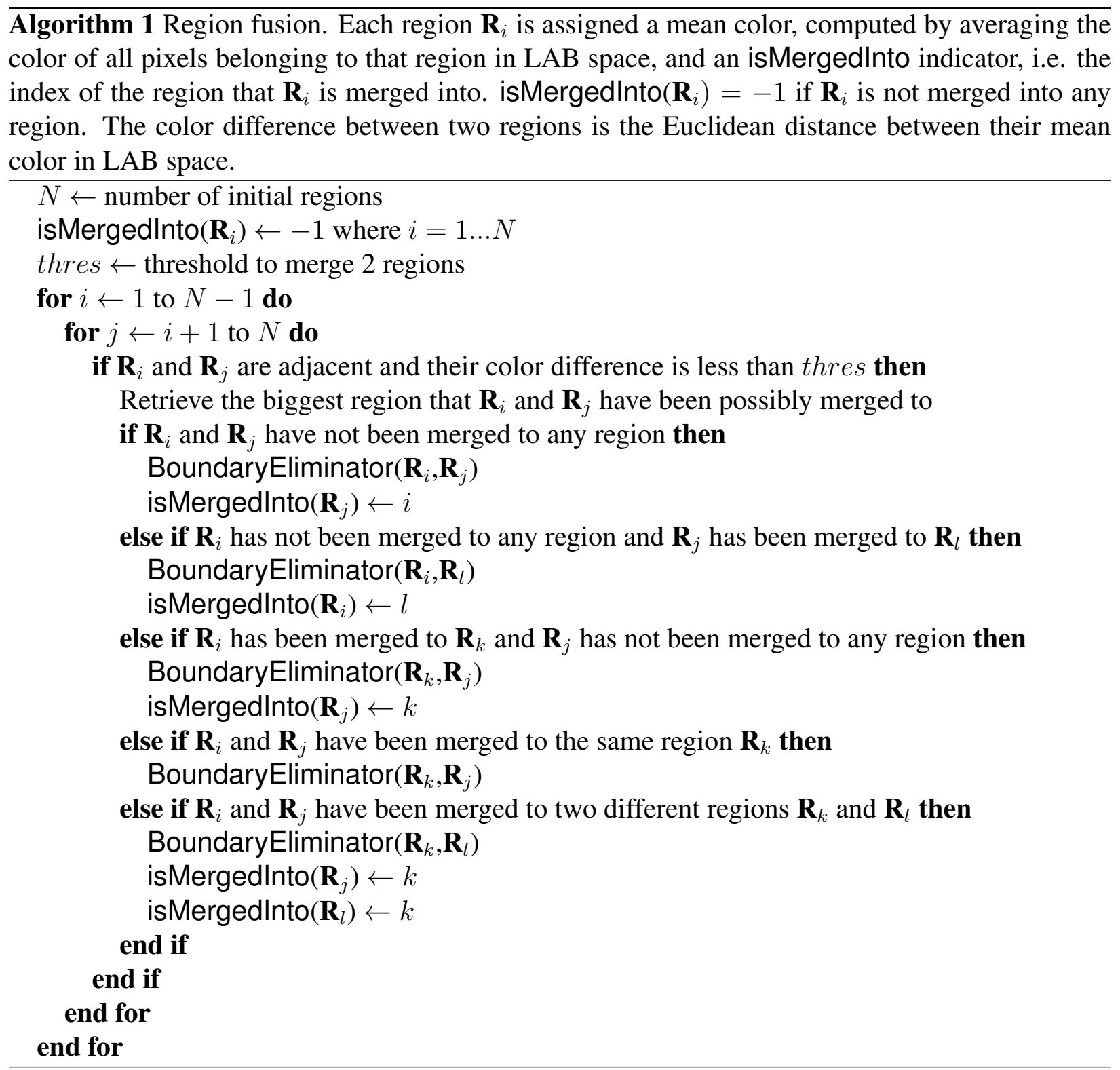




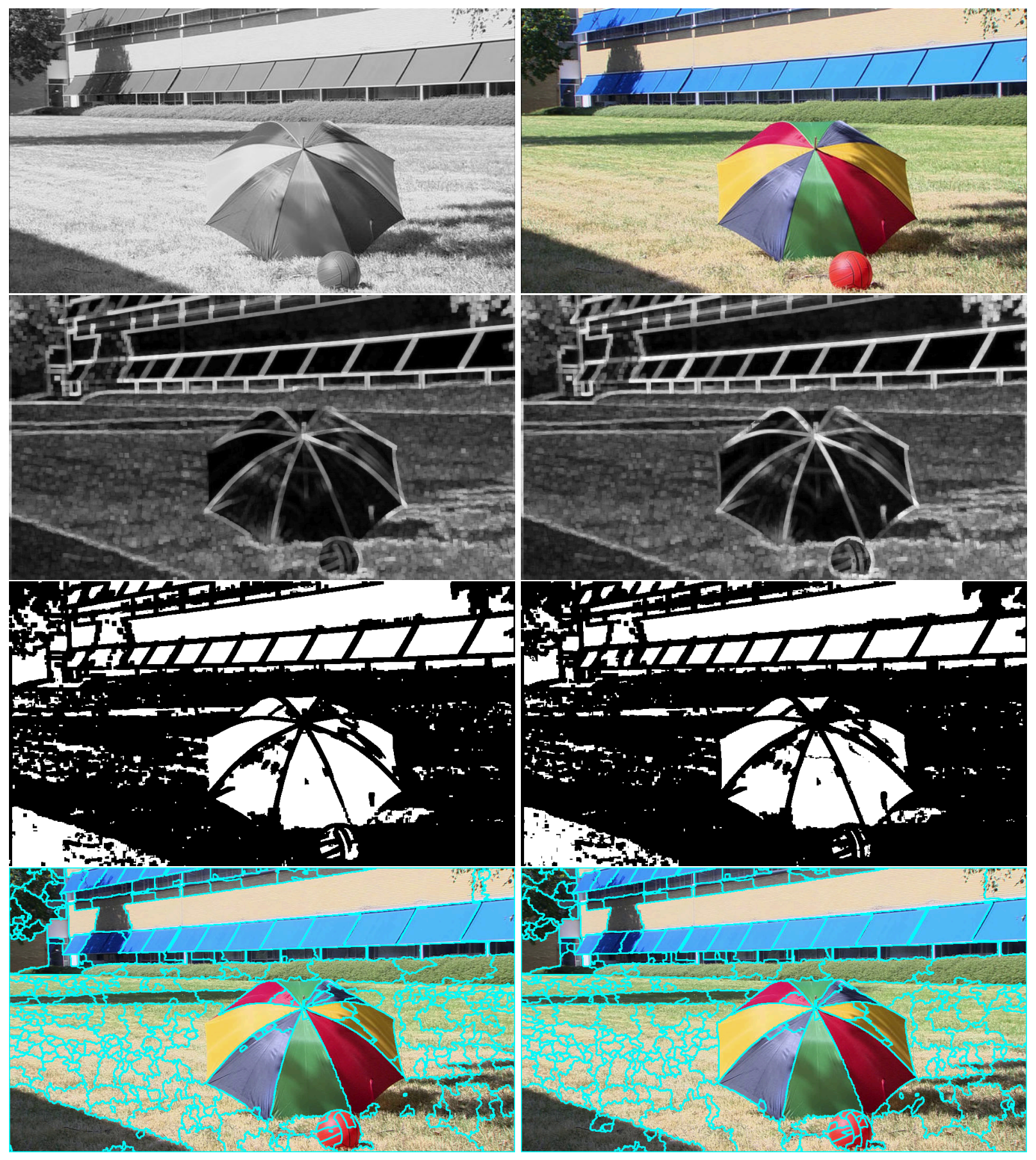

Fig 3 Watershed segmentation from the image gradient and markers. Row 1: input image in gray-level (left) and color (right). Row 2: image gradient from gray-level (left) and color (right) images. Row 3: markers computed from the image gradient and a threshold, post-processing such as opening/closing has been applied to refine markers. Row 4 : watershed segmentation. It can be seen that the color gradient preserves region boundaries better than the gray-level gradient. For instance, the outer edges of the umbrella with low contrast remains in the segmentation with the color gradient but disappears in the segmentation with the gray-level gradient. 

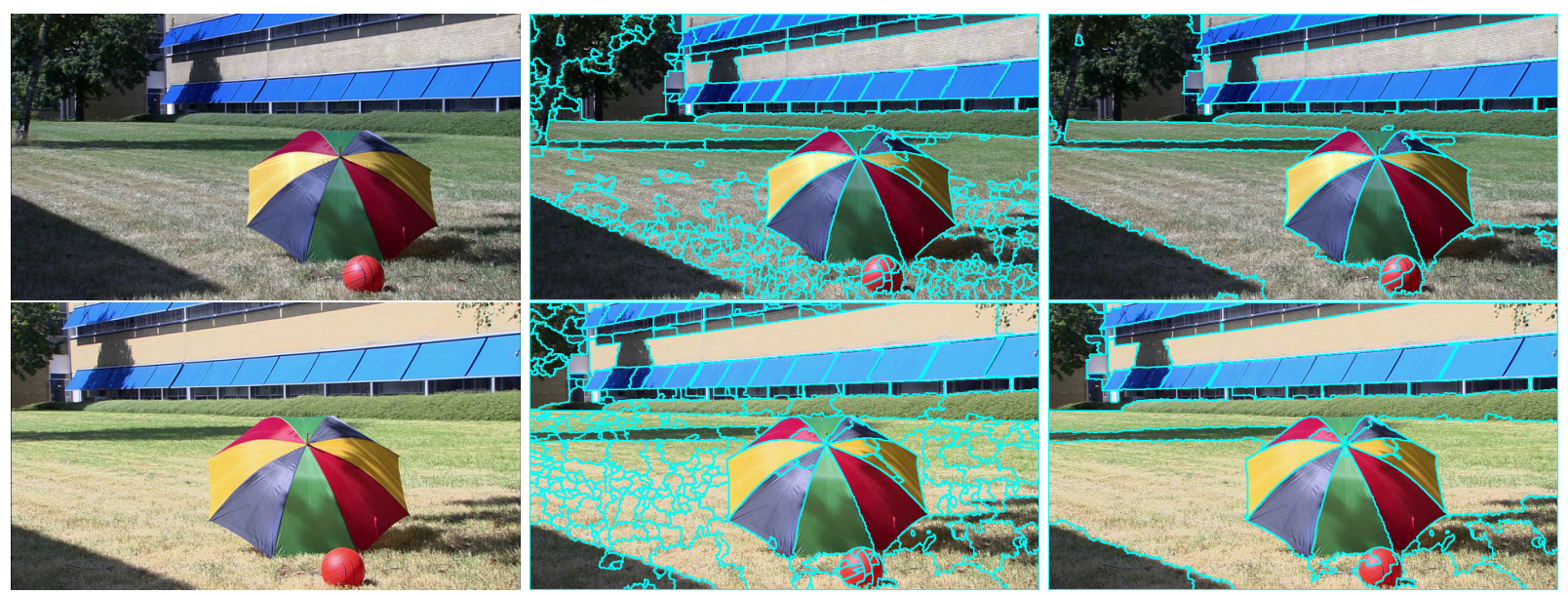

Fig 4 Region segmentation and fusion. Left: reference (upper) and input (lower) images. Middle: watershed segmentation. Right: region fusion. The watershed line is one-pixel large, but we increase their thickness for better visualisation.

\subsection{Region matching}

As the challenge is to search region correspondences between two images under severe geometric transformation and illumination change, we seek for an approach of region pairing using a criterion robust to these variations. We propose a method of region matching leveraged by image point features as follows

a. Compute point correspondences. Point features are detected and described using SIFT, 12 which is well known to be invariant to image translation, rotation, scaling and illumination change. Next, point correspondences are estimated using the Brute-Force matcher and refined by RANSAC ${ }^{13}$ with the fitting model being the homography between two images. The RANSAC is usually used to obtain robust data and the model best fitted to these data. In this case, we only require the robust point matches but do not employ the returned 2D homography. Note that we use RANSAC to obtain inliers but discard the output model. If we terminate the point matching here, the correspondences are the best matches fitted to the best estimated homography, therefore it is not guaranteed that they are spatially distributed 


\begin{tabular}{|c|c|c|c|}
\hline \multicolumn{2}{|c|}{ Reference image } & \multicolumn{2}{c|}{ Input image } \\
\hline Regions & Point correspondences & Point correspondences & Regions \\
\hline $\mathbf{R}_{i}$ & $\mathbf{p}_{1}$ & $\mathbf{p}^{\prime}$ & $\mathbf{R}^{\prime}{ }_{m}$ \\
\hline $\mathbf{R}_{j}$ & $\mathbf{p}_{2}$ & $\mathbf{p}^{\prime}$ & $\mathbf{R}^{\prime}{ }_{n}$ \\
\hline $\mathbf{R}_{k}$ & $\mathbf{p}_{3}$ & $\mathbf{p}^{\prime}$ & $\mathbf{R}{ }_{m}$ \\
\hline$\ldots$ & $\ldots$ & $\ldots$ & $\ldots$ \\
\hline $\mathbf{R}_{j}$ & $\mathbf{p}_{M}$ & $\mathbf{p}^{\prime}{ }_{M}$ & $\mathbf{R}^{\prime}$ \\
\hline
\end{tabular}

Table 1 Illustration of region matching leveraged by point correspondences

all over the image. In order to overcome this limited distribution, we implemented an incremental tiling approach: after one set of point correspondences is found, we mask the image part straddled by these points in both images and search for point correspondences within the unmasked image part. This mask can be computed by a rotated rectangle or a convex hull bounding a set of points. The point matching with mask is repeated until the final mask covers most of the image ( $80 \%$ in our experiments). Figure 5 illustrates the result of point matching by the incremental tiling technique and the region matching leveraged by these points.

b. Match two regions if they are straddled by matched points. Note that we discard points lying on the region borders (or the watershed lines). A simple illustration of this matching technique is presented in table 1. Regions $\mathbf{R}_{i}$ and $\mathbf{R}_{m}$ in the reference and input images respectively are matched as they contain matched points $\mathbf{p}_{1}$ and $\mathbf{p}_{1}{ }_{1}$. In addition, we merge regions in case of multiple-to-one matching, which may happen when several adjacent regions in one image correspond to a single region in the other image. For example, $\mathbf{R}_{i}$ and $\mathbf{R}_{k}$ are matched to $\mathbf{R}^{\prime}{ }_{m}$, hence $\mathbf{R}_{i}$ and $\mathbf{R}_{k}$ are merged. Similarly, $\mathbf{R}_{n}$ and $\mathbf{R}_{q}$ are merged as they are matched to the same region $\mathbf{R}_{j}$. 


\subsection{Color transfer}

The general color transfer function was introduced by Reinhard et al..$^{2}$ to scale and offset the color distribution of an input image (color distorted image) towards a reference image.

$$
\mathbf{C}_{\mathrm{o}}=\mu_{\mathrm{r}}+\frac{\sigma_{\mathrm{r}}}{\sigma_{\mathrm{i}}}\left(\mathbf{C}_{\mathrm{i}}-\mu_{\mathrm{i}}\right)
$$

where $\mathbf{C}_{\mathrm{i}}$ is the input image; $\mathbf{C}_{\mathrm{o}}$ is the output of color transfer; $\left(\mu_{\mathrm{i}}, \sigma_{\mathrm{i}}\right)$ and $\left(\mu_{\mathrm{r}}, \sigma_{\mathrm{r}}\right)$ are the (mean, standard deviation) of the input and reference images.

For each pair of regions $k$ found in the region matching step, we compute their color statistics $\mu_{\mathrm{i}}^{k}, \sigma_{\mathrm{i}}^{k}, \mu_{\mathrm{r}}^{k}$ and $\sigma_{\mathrm{r}}^{k}$, which will be the parameters of the color transfer between these two regions. Given $N$ region matches between the input and reference images, the color correction is a combination of $N$ local color transfers. In addition, in order to ensure a smooth color shading across the color-corrected image, each local color transfer is weighted by an influence mask, which measures the similarity of each pixel of the input image and the mean color of the region in consideration. 14

The influence mask of a region $k$ having the mean color $\mu_{\mathrm{i}}^{k}$ is generated as follows
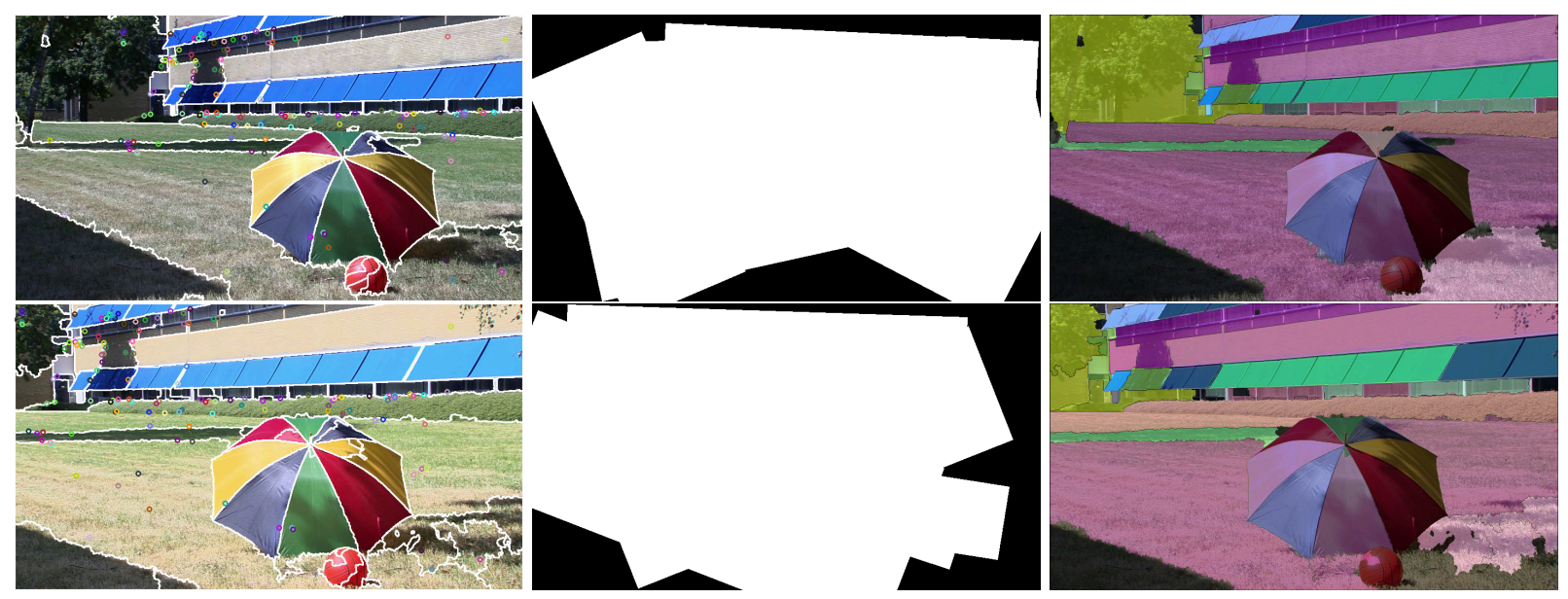

Fig 5 Region matching leveraged by point correspondences. Left: point correspondences by incremental tiling. Middle: the final masks bounding these points. Right: region correspondences in same color. 
a. The Euclidean distance between every pixel of the input image $\mathbf{C}_{\mathrm{i}}$ and the mean color of region $k$ in $\mathrm{LAB}$ space

$$
\mathbf{d}^{k}=\left\|\mathbf{C}_{\mathrm{i}}-\mu_{\mathrm{i}}^{k}\right\|
$$

b. In order to normalize the maximum of $\mathbf{d}$ which varies among different regions, we introduce the following distance bounded by 0 and 1

$$
\mathbf{p}^{k}=1-\frac{\mathbf{d}^{k}}{\max \left(\mathbf{d}^{k}\right)}
$$

An element of $\mathbf{p}$ approaches 1 when the color of the corresponding pixel in $\mathbf{C}_{\mathrm{i}}$ is close to $\mu_{\mathrm{i}}^{k}$ and 0 vice versa.

c. The influence mask

$$
\mathbf{I M}^{k}=e^{a\left(\mathbf{p}^{k}\right)^{b}}
$$

where $a$ and $b$ are tuning parameters. In our experiments, $a=10$ and $b=2$.

Figure 6 shows the influence masks calculated for three example regions.

The region-based color correction combines the color transfer functions of $N$ region correspondences weighted by $N$ influence masks

$$
\mathbf{C}_{\mathrm{o}}=\frac{\sum_{k=1}^{N}\left(\mu_{\mathrm{r}}^{k}+\frac{\sigma_{\mathrm{r}}^{k}}{\sigma_{\mathrm{i}}^{k}}\left(\mathbf{C}_{\mathrm{i}}-\mu_{\mathrm{i}}^{k}\right)\right) \times \mathbf{I M}^{k}}{\sum_{k=1}^{N} \mathbf{I} \mathbf{M}^{k}}
$$



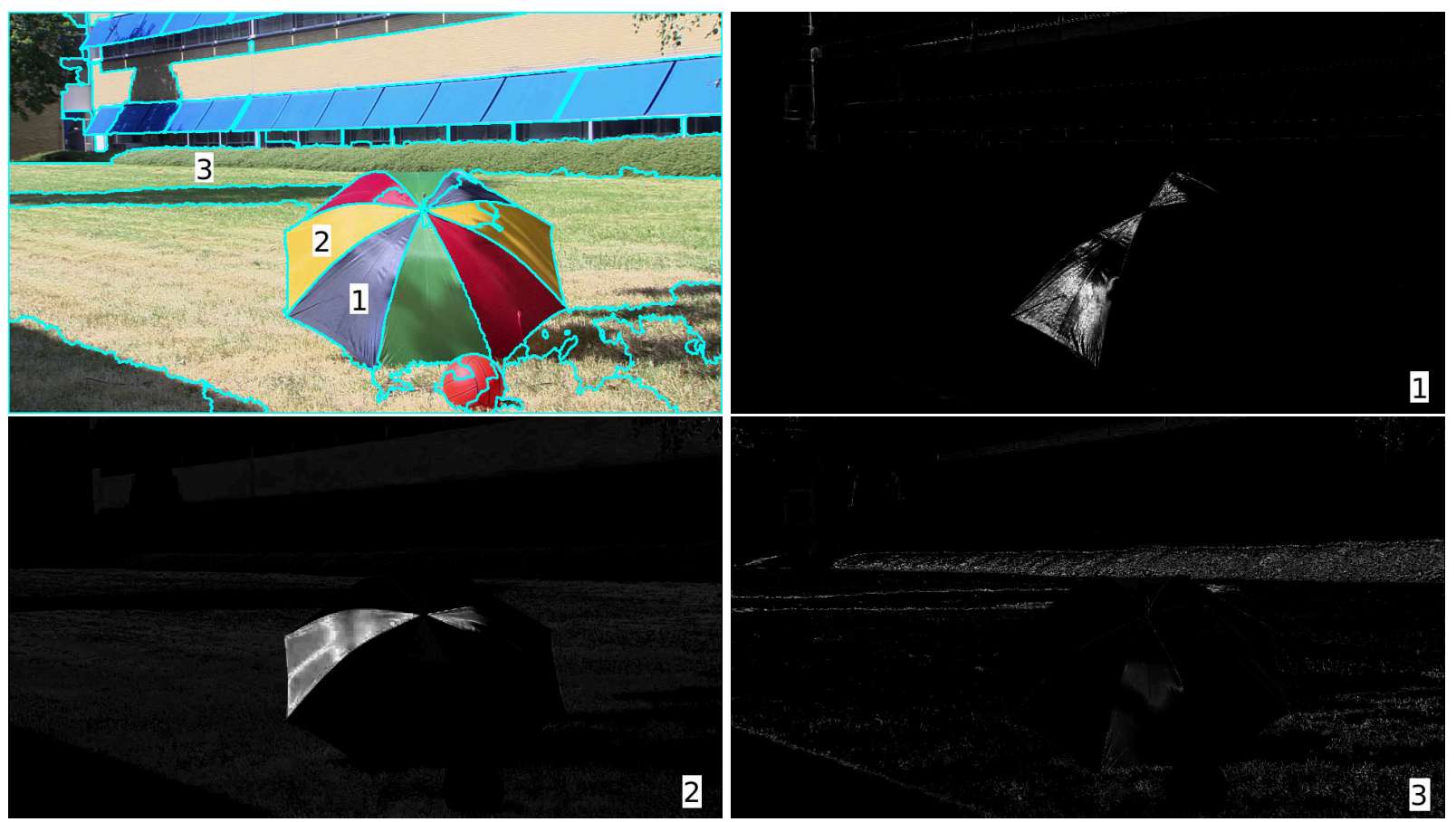

Fig 6 Three example regions of the input image and their corresponding influence masks. The closer the color of a pixel is to the mean color of the particular region, the higher its value in the influence mask of that region even though it is not within the region.

\section{Experiments}

\subsection{Data sets and evaluation metrics}

Three data sets are used to evaluate the color correction: "Football", "Desk" and "Umbrella". Each data set consists of a reference image, a color distorted image (which does not have the same content and color as the reference image) and two color corrected images (one by the global color correction $^{2}$ and the other by our proposed region-based color correction) as illustrated in figure 7 .

The color correction results are quantitatively evaluated by three metrics often used in image quality assessment: peak signal-to-noise ratio (PSNR), structural similarity index (SSIM) ${ }^{\sqrt{15}}$ and improved color image difference (iCID) ${ }^{\sqrt{16}}$ Their implementation is publicly available: PSNR and SSIM in the video quality measurement tool,,${ }^{17} \mathrm{iCID}$ in the supplementary material of ${ }^{16}$ We summarize the mechanism of these metrics as follows 
PSNR: The PSNR between two images A and B is defined by

$$
\operatorname{PSNR}(\mathbf{A}, \mathbf{B})=20 \log _{10} \frac{\max \mathbf{I}}{R M S E(\mathbf{A}, \mathbf{B})}
$$

where $\max \mathbf{I}$ is the highest possible pixel value of the image. Since each pixel is represented by 8 bits, $\max \mathbf{I}=255 . R M S E$ stands for the root mean square error. In this case, it is computed from all pixels of $\mathbf{A}$ and $\mathbf{B}$ in all color channels.

SSIM: Each pixel $x$ in image $\mathbf{x}$ consists of a lightness and two chromatic values $x=\left(L_{x}, a_{x}, b_{x}\right)$. Similarly for a pixel $y$ in image $\mathbf{y}, y=\left(L_{y}, a_{y}, b_{y}\right)$. The luminance difference $l(\mathbf{x}, \mathbf{y})$ is computed from the means of the lightness components $\mu_{x}$ and $\mu_{y}$. The contrast difference $c(\mathbf{x}, \mathbf{y})$ is measured from the standard deviations of the lightness components $\sigma_{x}$ and $\sigma_{y}$. The structure difference $s(\mathbf{x}, \mathbf{y})$ is the comparison of the normalised signals $\left(\mathbf{x}-\mu_{x}\right) / \sigma_{x}$ and $\left(\mathbf{y}-\mu_{y}\right) / \sigma_{y}$. Finally, these three differences are combined to yield an overall similarity measure $\operatorname{SSIM}(\mathbf{x}, \mathbf{y})=$ $f(l(\mathbf{x}, \mathbf{y}), c(\mathbf{x}, \mathbf{y}), s(\mathbf{x}, \mathbf{y}))$.

iCID: This measure is derived from the SSIM where as the differences in luminance, contrast and structure are computed from not only the lightness components $L_{x}$ and $L_{y}$ as in SSIM but also the chroma components $\left(a_{x}, b_{x}\right)$ and $\left(a_{y}, b_{y}\right)$. Three comparison terms are obtained separately and then multiplied by a factorial combination model to obtain the overall image difference measure.

These full-reference metrics measure the image similarity (or difference) between two images having the same content but different quality due to image/video compression, transmission errors, noise and blur, contrast or luminance changes. In real application of color correction, the reference and color distorted images have different content and color; we try thus to obtain the ground-truth image, which should have the same content as the color distorted image and the same color as the 
reference image. In short, the reference and color distorted images are used for the color correction whereas the ground-truth and color corrected images are used for the quantitative evaluation.

\subsection{Results}

"Umbrella" data set: The reference and color distorted images are captured by two cameras at different viewpoints and under the same illumination. The color rendered by these cameras is dissimilar due to their different photometric parameters. There is no ground-truth image, therefore there is no quantitative evaluation for this data set. By subjective perception, it can be seen that the global approach is erroneous. For example, the blue color (of the umbrella and the building outdoor blinds) and the yellow color (of the grass, the umbrella and the building wall) are not corrected properly. Since these images include non-corresponding regions, applying the global color transfer to the entire image produces incorrect results. On the contrary, the local technique provides good correction output.

"Football" and "Desk" data sets: For each data set, a sequence of images is captured by a single camera. Next, two arbitrary frames are selected: the first one is used as the reference image and the second one is modified in the gamma to obtain the color distorted image. The ground truth image is hence the second image before the gamma modification. The quantitative evaluation of the color correction applied to these data sets is presented in table 2, It can be seen that both global and region-based color correction approaches improve the color distorted image; and the regionbased method performs better than the global one. The reason why the performance of the global approach is quite good for these two image sets is that the reference and color distorted images contain very similar regions. 


\begin{tabular}{|c|c|c|c|c|}
\hline Data sets & Metrics & Gtr-Src & Gtr-GlobalCoCo & Gtr-LocalCoCo \\
\hline \multirow{3}{*}{ "Football" } & PSNR & 20.115126 & 24.355864 & 24.818874 \\
& SSIM & 0.796604 & 0.810221 & 0.813410 \\
& iCID & 0.5534 & 0.2460 & 0.2287 \\
\hline \multirow{3}{*}{ "Desk" } & PSNR & 22.480036 & 32.874046 & 35.191063 \\
& SSIM & 0.981346 & 0.986821 & 0.989409 \\
& iCID & 0.1159 & 0.0346 & 0.0258 \\
\hline
\end{tabular}

Table 2 Color correction quantitative results for the "Football" and "Desk" sets using three image quality metrics. The PSNR/SSIM measures the image similarity and the iCID measures the color difference between the ground truth image (Gtr) and each of the following: the color distorted image ( $\mathrm{Src}$ ), the color distorted image corrected by the global color correction (GlobalCoCo) or by the region-based color correction (LocalCoCo). The higher the value of PSNR/SSIM (and on the contrary, the lower the value of iCID), the more similar the two images in comparison.

\section{Conclusion and discussion}

We proposed an approach of region-based color correction. First, both reference and color distorted images are segmented to regions by rapid watershed transform. Then, regions between these images are paired using pre-extracted point correspondences which are invariant to geometric transformation and illumination variation. Finally, the weighted color transfer is applied to the color distorted image in order to modify its color distribution. One of the potential applications of this method can be found in television broadcasting to correct the color of video streams from various cameras, which may have different internal settings and external illumination conditions. A possible extension of this work is to develop an automatic color correction mechanism in which several color correction algorithms are integrated in a common framework and suitable image quality metrics are used to select the best color corrector from different correction results.

\section{Acknowledgments}

This work has been performed in the project PANORAMA, co-funded by grants from Belgium, Italy, France, the Netherlands, and the United Kingdom, and the ENIAC Joint Undertaking. 

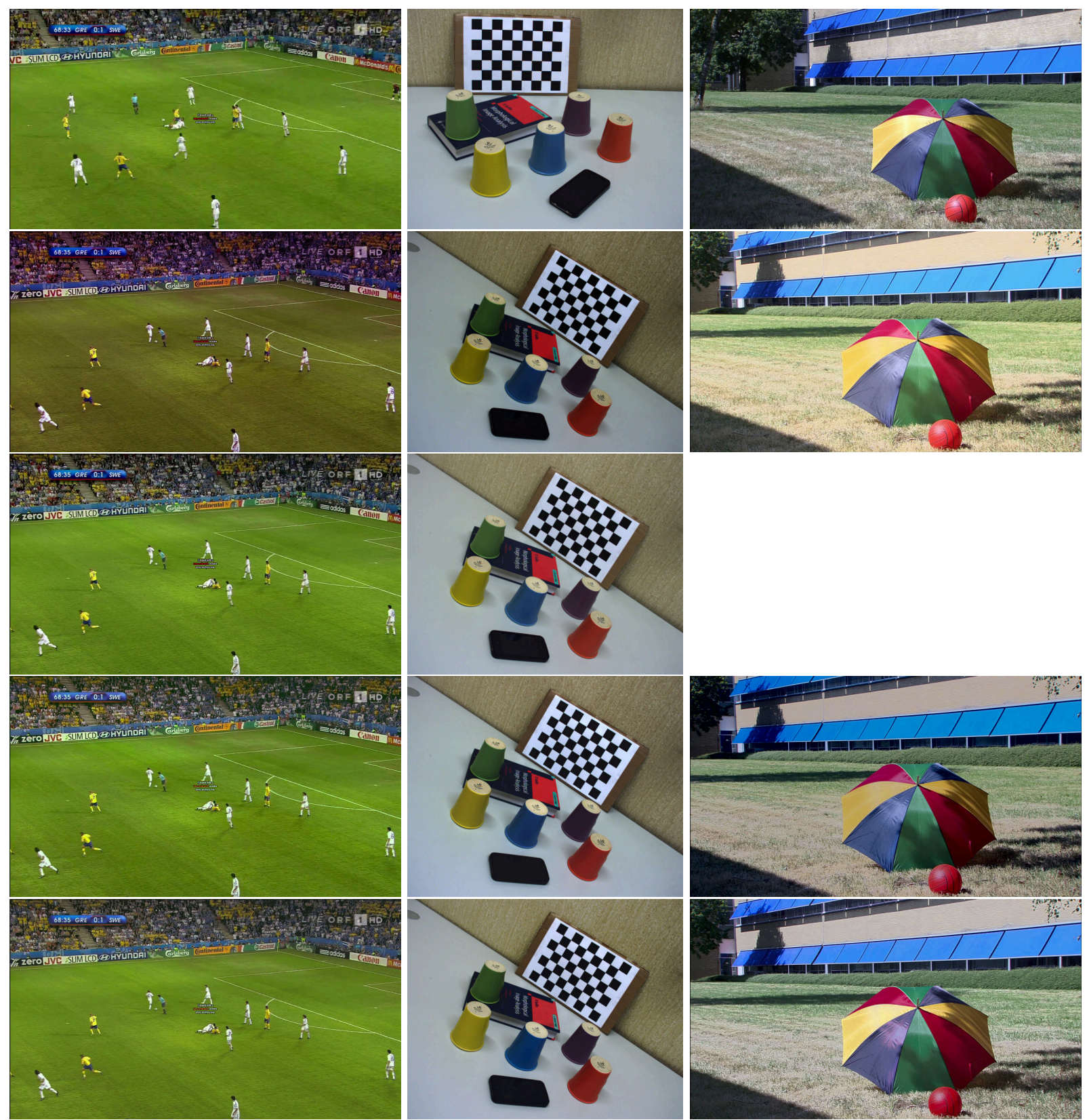

Fig 7 Color correction results on 3 data sets: "Football" (left), "Desk" (middle) and "Umbrella" (right). Row 1: reference image. Row 2: color distorted image. Row 3: ground truth image (only for "Football" and "Desk" sets). Row 4: global color correction.] Row 5: region-based color correction

\section{References}

1 F. M. Candocia and D. A. Mandarino, "A semiparametric model for accurate camera response function modeling and exposure estimation from comparametric data," IEEE Transactions on Image Processing 14(8), 1138-1150 (2005). 
2 E. Reinhard, M. Ashikhmin, B. Gooch, and P. Shirley, “Color transfer between images," IEEE Comput. Graph. Appl. 21, 34-41 (2001).

3 F. Pitie, A. C. Kokaram, and R. Dahyot, "N-dimensional probablility density function transfer and its application to colour transfer," in Proceedings of the Tenth IEEE International Conference on Computer Vision - Volume 2, ICCV '05, 1434-1439, IEEE Computer Society, (Washington, DC, USA) (2005).

4 R. C. Gonzalez and R. E. Woods, Digital Image Processing (3rd Edition), Prentice-Hall, Inc., Upper Saddle River, NJ, USA (2006).

5 S. Kagarlitsky, Y. Moses, and Y. Hel-Or, "Piecewise-consistent color mappings of images acquired under various conditions," in ICCV, 2311-2318, IEEE (2009).

6 Y.-W. Tai, J. Jia, and C.-K. Tang, "Local color transfer via probabilistic segmentation by expectation-maximization,” in $C V P R(1), 747-754$, IEEE Computer Society (2005).

7 M. Oliveira, A. D. Sappa, and V. Santos, "Unsupervised local color correction for coarsely registered images," in CVPR, 201-208 (2011).

8 W. Xu and J. Mulligan, "Performance evaluation of color correction approaches for automatic multi-view image and video stitching," in CVPR, 263-270 (2010).

9 R. Hartley and A. Zisserman, Multiple View Geometry in Computer Vision, Cambridge University Press, New York, NY, USA, 2nd ed. (2003).

10 D. S. Ly, S. Beucher, and M. Bilodeau, "Color correction through region matching leveraged by point correspondences," in International Conference on Image Processing, ICIP 2014, Paris, France, October 27-30, 2014, 640-644 (2014). 
11 S. Beucher and C. Lantuejoul, "Use of watersheds in contour detection," in International Workshop on Image Processing, (1979).

12 D. G. Lowe, "Object recognition from local scale-invariant features," in ICCV, 1150-1157 (1999).

13 M. A. Fischler and R. C. Bolles, "Random sample consensus: A paradigm for model fitting with applications to image analysis and automated cartography," Communications of the ACM 24, 381-395 (1981).

14 A. Maslennikova and V. Vezhnevets, "Interactive local transfer between images," in GraphiCon, 75-78 (2007).

15 Z. Wang, A. C. Bovik, H. R. Sheikh, and E. P. Simoncelli, "Image quality assessment: From error visibility to structural similarity," Trans. Img. Proc. 13, 600-612 (2004).

16 J. Preiss, F. Fernandes, and P. Urban, "Color-image quality assessment: From prediction to optimization," 23, 1366-1378 (2014).

17 P. Hanhart, "Video quality measurement tool."

Dieu Sang Ly is currently research engineer at Mines ParisTech, PSL - Research University. He received the B.Eng. degree in Electrical-Electronics Engineering from the University of Technology, Ho Chi Minh City, Vietnam in 2006; the M.Sc. degree in Computer Vision and Robotics from the Erasmus Mundus program in 2008 and the Ph.D. degree in Computer Vision from University of Picardie Jules-Verne, Amiens, France in 2011. His main research interests include camera modeling, image feature extraction, structure from motion and image processing with mathematical morphology. 
Serge Beucher, $\mathrm{PhD}$, is research director at PSL Research Institute (Paris School of Mines) and project leader for ARMINES. His professional interests have included segmentation techniques in image analysis, design of image analysers, development of morphological software on various platforms, real-time applications (on-board vision systems), industrial quality control by vision. His current research include image segmentation, computer vision, analysis of 3D scenes and image understanding.

Biographies and photographs of the other authors are not available.

\section{List of Figures}

$1 \quad$ Image segmentation

2 Region matching based on region color, location or known image registration

$3 \quad$ Watershed segmentation from the image gradient and markers

$4 \quad$ Region segmentation and fusion

$5 \quad$ Region matching leveraged by point correspondences

$6 \quad$ Influence masks for the color transfer

7 Color correction results

\section{List of Tables}

$1 \quad$ Illustration of region matching leveraged by point correspondences

2 Color correction quantitative results 